\title{
FDG-PET/CT activity leads to the diagnosis of unsuspected TB: a retrospective study
}

\author{
Carolina Geadas $^{1 *+}$, Carlos Acuna-Villaorduna ${ }^{1 \dagger}$, Gustavo Mercier ${ }^{2}$, Mary B. Kleinman³, \\ C. Robert Horsburgh Jr. ${ }^{1,4}$, Jerrold J. Ellner ${ }^{1}$ and Karen R. Jacobson ${ }^{1 *}$
}

\begin{abstract}
Objective: Mycobacterium tuberculosis infection leads to latent or active tuberculosis (TB). Increased uptake on ${ }^{18}$ F-fluoro-2-deoxy-glucose-positron emission tomography/computed tomography (FDG-PET/CT) has been reported in the lungs and lymph nodes of individuals with recent infection and active TB, but not in individuals without known recent exposure or suggestive symptoms. We describe five patients with lung nodules not suspected to be due to TB in whom abnormalities on FDG-PET/CT scans ultimately were attributed to TB infection.
\end{abstract}

Results: Patient records were searched using the words "positron emission tomography/computed tomography" and 24 codes for TB between 2004 and 2013. Patients with a diagnosis of TB and a PET/CT scan were included. Clinical and radiographic data were retrieved. PET/CT images were reviewed by an experienced radiologist. FDG-PET/CT scans revealed elevated FDG-uptake in lungs of five patients subsequently diagnosed with active $(n=3)$ or clinically inactive $(n=2)$ tuberculosis. Uptake magnitude was unrelated to disease activity. These findings suggest that tuberculosis latency may include periods of percolating inflammation of uncertain relationship to future disease risk.

Keywords: Latent, Subclinical TB, Imaging, Biomarkers, Risk

\section{Introduction}

The idea that Mycobacterium tuberculosis (MTB) infection exists in two discrete states-metabolically-inactive latent tuberculosis infection (LTBI) and metabolicallyactive tuberculosis (TB) disease-has recently been challenged with a new model of fluctuating activity ranging from quiescent to metabolically-active lesions $[1,2]$. The American Thoracic Society (ATS) classifies persons exposed to and/or infected with MTB into distinct classes: [3] Class 1 comprises individuals with a history of exposure to TB but no evidence of infection; Class 2 includes individuals with evidence of LTBI but not of active disease; persons with Class 3 have clinically active disease; and Class 4 includes those with either a history of previous clinically active TB or a history of exposure to

\footnotetext{
*Correspondence: carolinageadas@gmail.com; karen.jacobson@bmc.org ${ }^{\dagger}$ Carolina Geadas and Carlos Acuna-Villaorduna contributed equally to this work

1 Section of Infectious Diseases, Department of Medicine, Boston Medical Center and Boston University School of Medicine, 801 Massachusetts Ave, 2nd Floor, Boston, MA 02118, USA

Full list of author information is available at the end of the article
}

TB with abnormal stable findings on chest radiography, without clinically active disease.

${ }^{18}$ F-fluoro-2-deoxy-glucose-positron emission tomography/computed tomography (FDG-PET/CT) is a noninvasive imaging tool used primarily for cancer diagnosis and staging. It identifies areas of active inflammation by mapping where cells with high metabolic demand take up the radioactively-labeled glucose analogue (FDG). The radiotracer accumulates within inflammatory cells such as macrophages and neutrophils and can be quantified as a standardized uptake value (SUV). Previous reports have shown increased FDG-uptake in the lungs and lymph nodes of persons presumed to be recently infected with TB (household contacts of TB cases with a positive QuantiFERON-TB Gold) [4], in asymptomatic HIV coinfected individuals in a high TB burden setting [5], and in individuals with active TB lesions [6, 7]. FDG-uptake has variably risen or fallen in response to treatment [7]. Previous studies have investigated activity in persons with recent or ongoing TB exposure or in the process of TB disease diagnosis or treatment [8]. We identified five patients with TB infection [per positive tuberculin skin

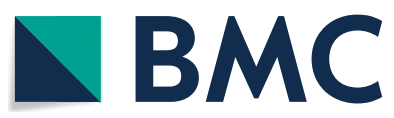

(c) The Author(s) 2018. This article is distributed under the terms of the Creative Commons Attribution 4.0 International License (http://creativecommons.org/licenses/by/4.0/), which permits unrestricted use, distribution, and reproduction in any medium, provided you give appropriate credit to the original author(s) and the source, provide a link to the Creative Commons license, and indicate if changes were made. The Creative Commons Public Domain Dedication waiver (http://creativecommons.org/ publicdomain/zero/1.0/) applies to the data made available in this article, unless otherwise stated. 
test (TST) or interferon-gamma release assay (IGRA)], but not suspected to have TB disease, that underwent FDG-PET/CT scan for evaluation of pulmonary nodules and had the final pathologic and/or microbiologic diagnoses of TB.

\section{Main text \\ Methods}

This retrospective study was conducted at Boston Medical Center (BMC) - a safety-net hospital with a large immigrant patient population. We searched the BMC clinical database using the words "positron emission tomography/computed tomography" in combination with 24 ICD-9 codes for TB. Patients that had both a FDG-PET/CT scan and a TB diagnosis were included if: (1) results were between January 2004 and December 2013 and (2) a diagnosis of Class 3 or 4 TB (per ATS standard classification [3]) was made within 90 days of a FDG-PET/CT. Medical records of included patients were abstracted. FDG-PET/CT images were reviewed by a nuclear radiologist (GM) who was blinded to the diagnoses. The study was approved by the BMC Institutional Review Board (IRB).

\section{Results}

We identified 159 patients whose medical records included both a FDG-PET/CT scan and TB ICD-9 code. One hundred fifty-two were excluded: eight had a TB disease diagnosis prior to the scan, eight had only a history of TB exposure without evidence of infection (Class 1 TB), and 136 had a history of LTBI with no evidence of clinically active TB disease (Class 2 TB) - these individuals received alternative diagnoses for their pulmonary nodules.

We identified seven patients whose FDG-PET/CT scans showed pulmonary abnormalities that were ultimately attributed to TB. This included three cases of culture-proven TB (Class 3 ) and four cases of inactive TB (Class 4) who had evidence of LTBI and abnormal findings on PET/CT, but no clinical, microbiological or radiographic evidence of active disease. For two of the patients identified as having Class 4, wedge resections were not performed and therefore a diagnosis could not be unequivocally confirmed by histopathology. For this reason, these two patients were excluded from this evaluation.

The three patients with culture-positive active (Class 3) TB (Table 1) were males from high TB burden countries (immigrated to the US in 1988, 1998, and 2010). Mean age was 55 years (range 34-77). One patient was HIV-infected (CD4 $=26$ cubic cells per millimeter, not on antiretrovirals). Two had symptoms consistent with TB (cough, weight loss, night sweats) and one had had a selfresolved respiratory infection. All three patients showed abnormal uptake by upper lobe nodules on lung PET/

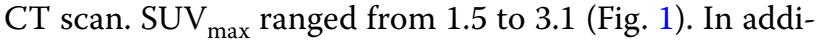
tion to pulmonary $\mathrm{TB}$, one patient had extra-pulmonary disease confirmed by a positive MTB culture of a colonic ulceration. In all three patients, TB was microbiologically confirmed by positive microscopy, culture, and/or polymerase chain reaction (PCR) of the sputum or bronchoalveolar lavage (Table 1).

The two individuals with culture-negative, inactive (Class 4) TB (Table 1) were both males from high TB burden countries as well (immigrated to the US in 2004 and 2010). Mean age was 63.5 years (range 58-69). Both patients had diabetes. Both were asymptomatic. On PET/ CT scan, both showed increased FDG-uptake by upper

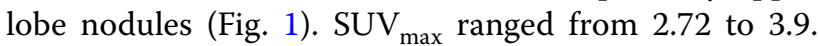
Neither had clinical evidence of extra-pulmonary disease by symptoms or on PET/CT. Both had negative sputum microscopies and cultures. Wedge resection of the areas with increased uptake was performed and in both cases necrotizing granulomas were identified on histopathology; this tissue was also negative for acid fast bacilli (AFB) smears and mycobacterial cultures did not grow (Table 1).

All five patients were subsequently treated with isoniazid and rifampin or with a four-drug regimen of isoniazid, rifampin, pyrazinamide, and ethambutol per standard ATS guidelines.

\section{Discussion}

Although increased PET activity has been described in cases of active TB and recent TB infection, the finding of increased activity in asymptomatic individuals without recent exposure has not been reported. We found two asymptomatic LTBI patients who had wedge resections in the region of increased FDG-uptake that revealed granulomas, indicating that $\mathrm{TB}$ was driving the metabolic activity. The presence of metabolic activity in individuals without symptoms or known recent TB exposure supports the concept that TB latency is not static; metabolic activity may fluctuate during latency driven by a lowlevel replicating mycobacterial burden as demonstrated by our patients' negative cultures and lack of constitutional symptoms. It is unclear whether in the absence of treatment these foci would regress spontaneously or progress to active disease. Autopsy studies, in fact, have revealed evidence of minimally active $\mathrm{TB}$ in patients that died from other causes and still were asymptomatic [2]. A lesson of HIV infection is that viable organisms exist within latent foci and have the potential to reactivate when no longer suppressed [9]. Cell wall synthesis of bacilli also must occur during latency as isoniazid, a bactericidal cell-wall active drug that acts on replicating 


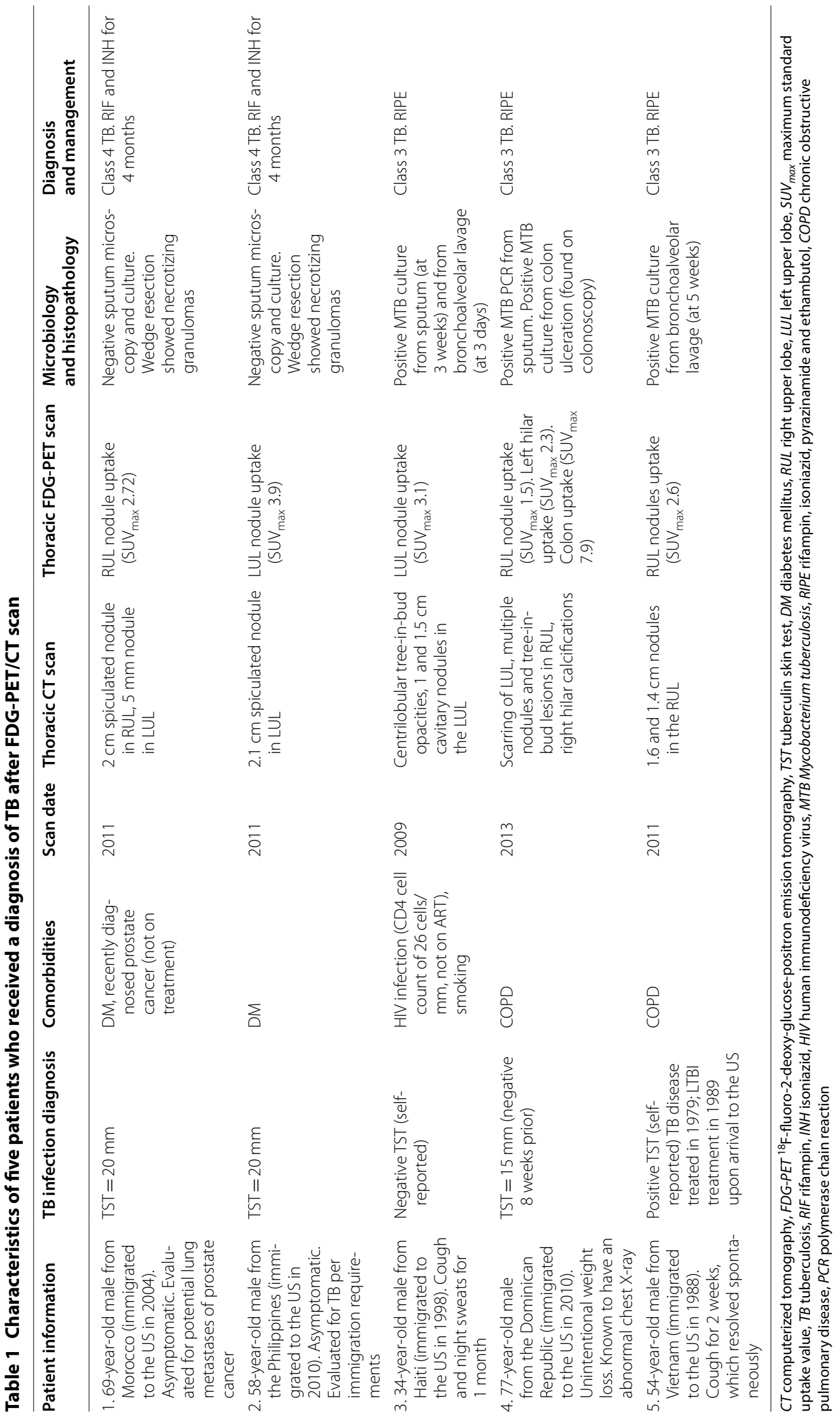



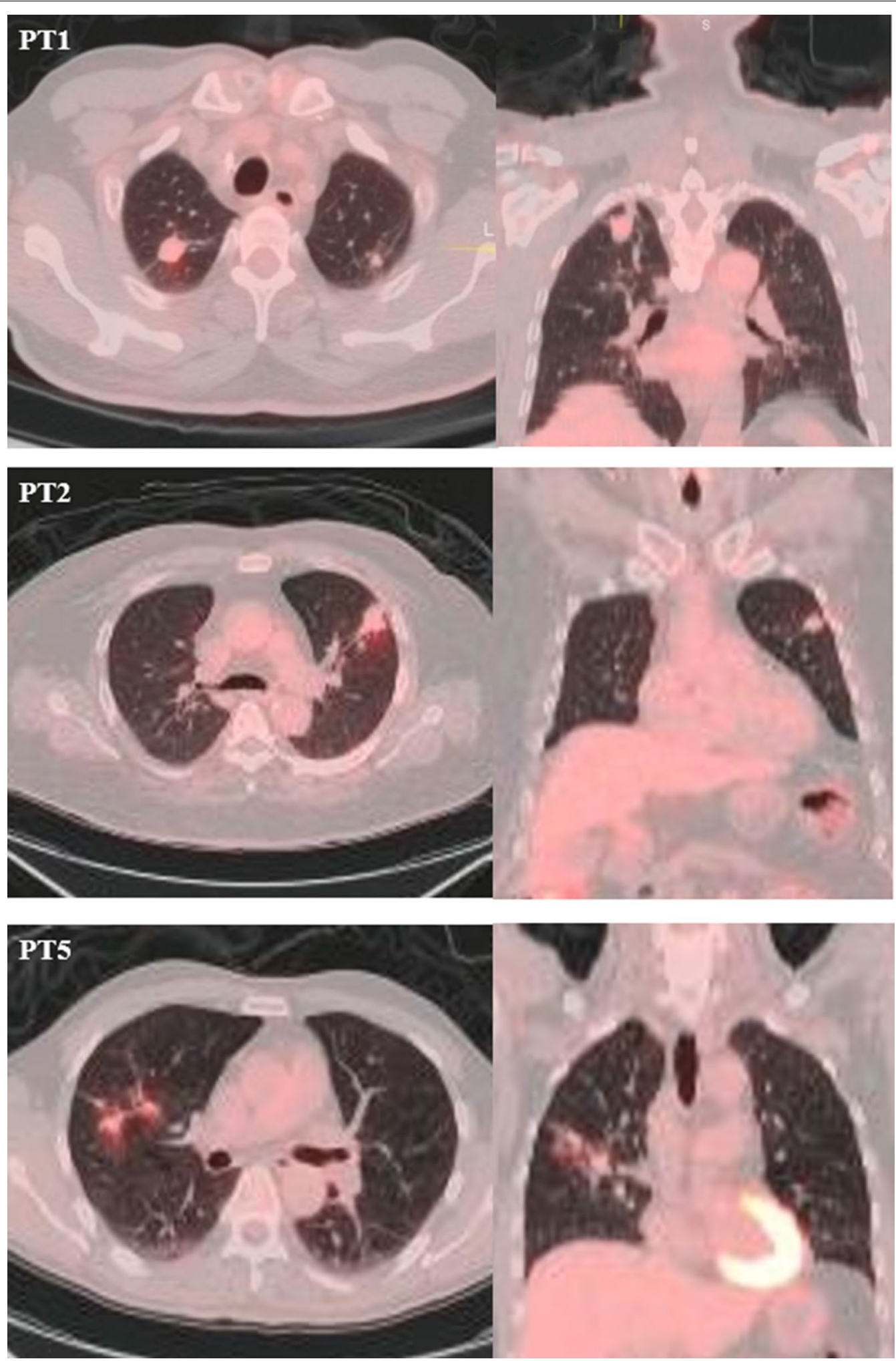

Fig. 1 FDG-PET/CT scans of patients (PT) 1, 2, and 5. Images are axial and coronal fused PET/CT images with CT in standard lung windows and the PET in an orange color scale. PT1 shows a $2 \mathrm{~cm}$ spiculated lung nodule in the right upper lobe (RUL) with SUV max $_{\text {Of }} 2.72$. PT2 shows a similar finding in the left upper lobe (LUL) but tracer uptake is slightly higher with SUV $\max$ of 3.9. PT5 shows two RUL nodules, with 1.6 and $1.4 \mathrm{~cm}$, both with SUV $V_{\max }$ of 2.6 
bacilli, successfully prevents progression from LTBI to TB disease [2].

With its high sensitivity, PET/CT captures activity reflecting very low burden TB. A recent study reported that among HIV co-infected individuals with LTBI, those with lung abnormalities on FDG-PET/CT (including infiltrates, fibrotic scars, or active nodules) were more likely to subsequently develop symptomatic active disease than those without [5]. Interestingly the two asymptomatic patients identified in our study (patients 1 and 2) had $S U V_{\text {max }}$ in the same range (2.7-3.9) as the three symptomatic patients (patients 3,4 , and 5) who had culturable TB (range 1.5-3.1), suggesting that these groups may be very close in the disease spectrum. It is notable, although difficult to explain, that despite similar inflammation the Class 3 cases were symptomatic whereas the Class 4 cases were not. A current study investigating the natural history of FDG-avid pulmonary nodules in HIV-uninfected contacts of multidrug-resistant TB cases in Cape Town, South Africa (DMID protocol number 16-0112), should shed light on the rate of progression to active disease.

\section{Conclusions}

Combined with our findings, the current studies indicate that symptom screening and sputum smear and mycobacterial culture are limited in their ability to detect TB activity, including latency. Improved biomarkers are needed to stratify individuals according to risk of progression to active disease. FDG-PET/CT may reflect an early event in TB reactivation and could be of value for biomarker development. Our observations raise two important points in clinical practice. The first is that $\mathrm{TB}$ may produce very similar findings to lung cancer on FDG-PET/CT, making it essential to keep TB in the differential diagnosis even in patients without a known recent exposure or classic symptoms. The second is that, in patients with remote exposure to $\mathrm{TB}$ or a known history of LTBI (per positive TST or IGRA), FDG-PET/CT activity may reflect low-burden TB disease with imminent reactivation, prompting treatment.

\section{Limitations}

A limitation of our study is that none of our patients had follow-up PET/CT scans to determine whether FDG activity resolved after treatment. In a series of recently infected persons, increased lymph node activity diminished in three of four patients after treatment of LTBI [4]. Additionally, quantitative changes in FDG-uptake two months after starting treatment were associated with long term outcomes in a cohort of multidrug-resistant TB patients receiving treatment in South Korea [10]. On the other hand, most HIV-uninfected patients with drug-sensitive TB treated in South Africa had persistent $\mathrm{PET} / \mathrm{CT}$ activity at the time of sputum culture conversion and even up to one year after [11]. It is therefore unclear whether the foci of FDG activity described in our study would have regressed spontaneously or progressed to active disease without treatment.

\section{Abbreviations \\ ATS: American Thoracic Society; CT: computerized tomography; FDG-PET: ${ }^{18} \mathrm{~F}$-fluoro-2-deoxy-glucose-positron emission tomography; HIV: human immunodeficiency virus; IGRA: interferon-gamma release assay; LTBI: latent tuberculosis infection; PCR: polymerase chain reaction; SUV: standard uptake value; TB: tuberculosis; TST: tuberculin skin test.}

\section{Authors' contributions}

CG and CA participated in study conception and design, data analysis and interpretation, and manuscript drafting. GM reviewed and interpreted PET/CT imaging and participated in manuscript review. MK aided in study conception and design as well as data collection and review. JE and RH worked on study conception and design in addition to manuscript drafting and review. $\mathrm{KJ}$ helped conceive and design the study as well as collecting data, reviewing and interpreting the data; KJ also drafted and reviewed the manuscript. All authors read and approved the final manuscript.

\section{Author details \\ ${ }^{1}$ Section of Infectious Diseases, Department of Medicine, Boston Medical Center and Boston University School of Medicine, 801 Massachusetts Ave, 2nd Floor, Boston, MA 02118, USA. ${ }^{2}$ Section of Molecular Imaging and Nuclear Medicine, Department of Radiology, Boston Medical Center and Boston Uni- versity School of Medicine, Boston, MA, USA. ${ }^{3}$ Infectious Disease Prevention and Health Services Bureau, Prevention and Health Promotion Administration, Maryland Department of Health and Mental Hygiene, Baltimore, MD, USA. ${ }^{4}$ Department of Epidemiology, Boston University School of Public Health, Boston, MA, USA.}

\section{Acknowledgements}

The authors would like to thank Elizabeth Ragan, MPH for her administrative support to this work.

\section{Competing interests}

The authors declare that they have no competing interests.

\section{Availability of data and materials}

All data generated or analyzed during this study are included in this published article.

\section{Consent for publication \\ Not applicable. All identifiers were removed from the case reports.}

\section{Ethics approval and consent to participate}

This study was approved by the Boston Medical Center (BMC) Institutional Review Board (IRB). Given the retrospective nature of this study and the very low risk for its participants, the need for informed consent was waived by the BMC IRB. Records were accessed under consent from the hospital administration.

\section{Funding}

Research reported in this publication was supported by the National Institute of Allergy and Infectious Diseases of the National Institutes of Health under Award Number U19Al111276. The content is solely the responsibility of the authors and does not necessarily represent the official views of the National Institutes of Health.

\section{Publisher's Note}

Springer Nature remains neutral with regard to jurisdictional claims in published maps and institutional affiliations. 
Received: 17 April 2018 Accepted: 5 July 2018

Published online: 13 July 2018

\section{References}

1. Salgame P, Geadas C, Collins L, Jones-López E, Ellner JJ. Latent tuberculosis infection-revisiting and revising concepts. Tuberculosis (Edinb). 2015;95:373-84. https://doi.org/10.1016/j.tube.2015.04.003.

2. Dutta NK, Karakousis PC. Latent tuberculosis infection: myths, models, and molecular mechanisms. Microbiol Mol Biol Rev. 2014;78:343-71. https://doi.org/10.1128/MMBR.00010-14.

3. American Thoracic Society. Diagnostic standards and classification of tuberculosis in adults and children. Am Thorac Soc Am J Respir Crit Care Med. 2000;161:1376-95.

4. Ghesani N, Patrawalla A, Lardizabal A, Salgame P, Fennelly KP. Increased cellular activity in thoracic lymph nodes in early human latent tuberculosis infection. Am J Respir Crit Care Med. 2014;189:748-50. https://doi. org/10.1164/rccm.201311-1976LE.

5. Esmail H, Lai RP, Lesosky M, Wilkinson KA, Graham CM, Coussens AK, et al. Characterization of progressive HIV-associated tuberculosis using 2-deoxy-2-[18F]fluoro-D-glucose positron emission and computed tomography. Nat Med. 2016;22:1090-3. https://doi.org/10.1038/nm.4161.
6. Ankrah AO, van der WerfTS, de Vries EFJ, Dierckx RAJO, Sathekge MM, Glaudemans AWJM. PET/CT imaging of Mycobacterium tuberculosis infection. Clin Transl Imaging. 2016;4:131-44. https://doi.org/10.1007/s4033 6-016-0164-0.

7. Skoura E, Zumla A, Bomanji J. Imaging in tuberculosis. Int J Infect Dis. 2015;32:87-93. https://doi.org/10.1016/j.ijid.2014.12.007.

8. Heysell SK, Thomas TA, Sifri CD, Rehm PK, Houpt ER. 18-Fluorodeoxyglucose positron emission tomography for tuberculosis diagnosis and management: a case series. BMC Pulm Med. 2013;13:14. https://doi. org/10.1186/1471-2466-13-14.

9. Pinkevych M, Cromer D, Tolstrup M, Grimm AJ, Cooper DA, Lewin SR, et al. HIV reactivation from latency after treatment interruption occurs on average every 5-8 days - implications for HIV remission. PLoS Pathog. 2015;11:e1005000. https://doi.org/10.1371/journal.ppat.1005000.

10. Chen RY, Dodd LE, Lee M, Paripati P, Hammoud DA, Mountz JM, et al. PET/ CT imaging correlates with treatment outcome in patients with multidrug-resistant tuberculosis. Sci Transl Med. 2014. https://doi.org/10.1126/ scitranslmed.3009501

11. Malherbe ST, Shenai S, Ronacher K, Loxton AG, Dolganov G, Kriel M, et al. Persisting positron emission tomography lesion activity and Mycobacterium tuberculosis mRNA after tuberculosis cure. Nat Med. 2016;22:1094100. https://doi.org/10.1038/nm.4177.
Ready to submit your research? Choose BMC and benefit from:

- fast, convenient online submission

- thorough peer review by experienced researchers in your field

- rapid publication on acceptance

- support for research data, including large and complex data types

- gold Open Access which fosters wider collaboration and increased citations

- maximum visibility for your research: over $100 \mathrm{M}$ website views per year

At BMC, research is always in progress.

Learn more biomedcentral.com/submissions 